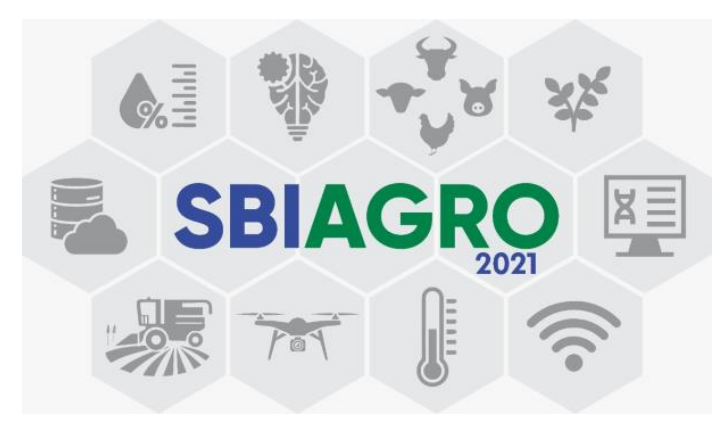

\title{
Desempenho da classificação supervisionada com a inclusão da banda termal em áreas selecionadas do bioma Pampa - Brasil
}

\section{Supervised classification performance with the inclusion of the thermal band in selected areas of the Pampa biome - Brazil}

\section{Patricia Michele Pereira Trindade ${ }^{1,}$ Daniela Wancura Barbieri Peixoto ${ }^{1}$, Tatiana Mora Kuplich ${ }^{1}$, Claudio Aparecido de Almeida ${ }^{2}$}

${ }^{1}$ Instituto Nacional de Pesquisas Espaciais (INPE) - Coordenação Espacial do Sul (COESU), Santa Maria - RS

${ }^{2}$ Divisão de Projetos Estratégicos 1 (DIPE1), São José dos Campos - SP, Brasil

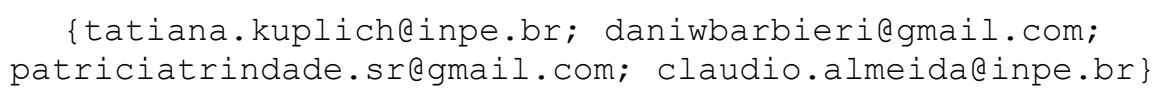

\begin{abstract}
The Pampa biome is the focus of profound changes in recent decades and grasslands have been converted to rice, soybean and forestry crops. In this article, it was investigated whether the addition of the thermal band in Maxver supervised classification with Landsat images increased the accuracy of vegetation cover mappings in the Pampa Biome. The results pointed out to a moderate increase in accuracy of the classifications with the use of the thermal band. Visual interpretation of images is also facilitated with temperature data. For both approaches (digital and manual), it is important to consider seasonal images, which include vegetation in different stages and vigor conditions.
\end{abstract}

Resumo. O bioma Pampa é foco de profundas alterações nas últimas décadas e os campos nativos têm sido convertidos para culturas de arroz, soja e silvicultura. Neste artigo foi investigada se a adição de banda do termal em classificação supervisionada Maxver com imagens Landsat aumentaria a acurácia dos mapeamentos de cobertura vegetal no Bioma Pampa. Os resultados apontam para moderado aumento na acurácia das classificações com a utilização da banda do termal. A interpretação visual das imagens também é facilitada com dados de temperatura. Para ambas abordagens 
(digital e manual), é importante imagens temporais de épocas distintas, que contemplem coberturas vegetais em diferentes estágios e condições de vigor.

\section{Introdução}

O bioma Pampa ocupa 63\% do território do Rio Grande do Sul e é uma das áreas de campos temperados mais importante do mundo (MMA, 2019). O Pampa é um dos biomas brasileiros que teve sua cobertura vegetal, primariamente campestre, bastante alterada (Embrapa, 2016), e parte destas alterações são devidas à expansão das áreas de cultivo agrícola e silvicultural (Nabinger et al. 2009). Na metade sul do RS a principal ocupação do bioma sempre foi a pecuária extensiva, porém a partir do século $\mathrm{XX}$ ocorreu a implantação da agricultura intensiva, destacando a produção de arroz irrigado nas áreas de várzeas. No início do século XXI começou a elevada expansão da silvicultura e cultivo de soja (MMA, 2019).

Neste cenário de conversões de cobertura da terra, dados de sensoriamento remoto se revelam essenciais para a detecção e mapeamento dos campos e de seu estado de conservação, como no Programa de Monitoramento Ambiental dos Biomas Brasileiros, do Ministério do Meio Ambiente, executado por equipes do INPE e FUNCATE (Fundação de Ciência, Aplicações e Tecnologia Espaciais ), cujos dados estão disponíveis na Plataforma Terrabrasilis - http://terrabrasilis.dpi.inpe.br/ (Assis et al., 2019, Almeida, 2020).

A utilização de bandas na faixa do termal em associação às bandas ópticas tem sido apontada como favorável à discriminação de coberturas da terra (Rodriguez-Galiano et al. 2012, Sun e Schulz 2015, Eisavi et al. 2015, Zhao et al. 2019). O aumento da acurácia de classificações automáticas, mesmo supervisionadas (com treinamento), em mapeamentos de coberturas vegetais, poderá contribuir para o monitoramento da conservação e manejo dos biomas brasileiros.

Este artigo teve como objetivo avaliar a adição da banda termal (banda 10) do sensor TIRS (Thermal Infrared Sensor) às bandas ópticas OLI (Operational Land Imager) do satélite Landsat 8 na classificação de campos e outras coberturas vegetais do bioma Pampa, com a abordagem de Máxima Verossimilhança (Maxver).

\section{Metodologia}

Para a realização das etapas metodológicas, selecionou-se nove células de 25 x $25 \mathrm{~km}$ no bioma Pampa (Figura 1). As células foram distribuídas nas regiões leste, centro e oeste nas órbitas ponto 221/81, 223/81 e 224/81 respectivamente. 


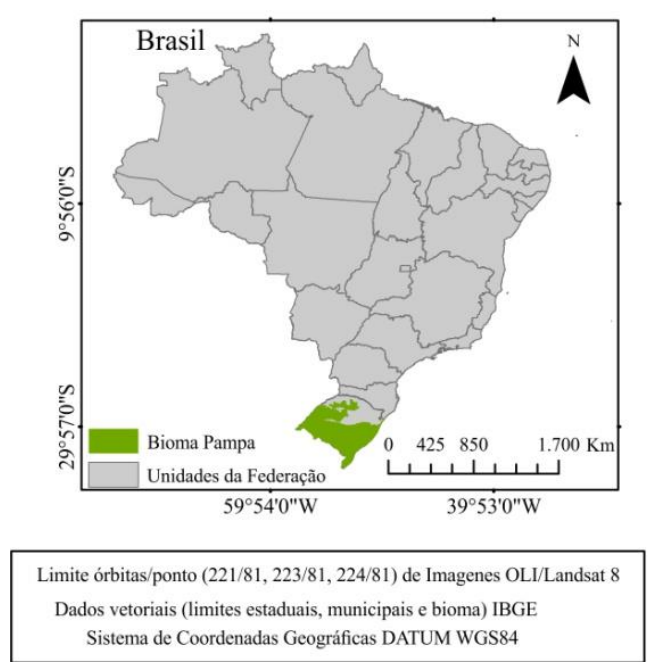

stema de Coordenadas Geográficas DATUM WGS84

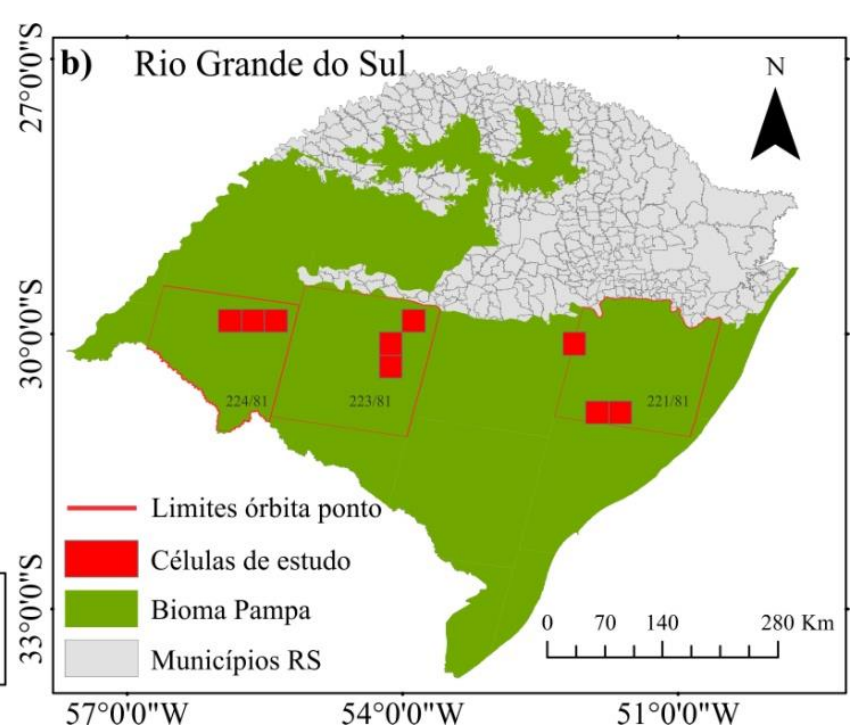

Figura 1. - Localização e limites do Bioma Pampa, das células classificadas e das órbitas ponto das imagens Landsat utilizadas no estudo.

\subsection{Materiais}

Foram utilizadas imagens dos sensores OLI e TIRS do satélite Landsat 8 , correspondentes às datas 15/02/2018, 26/08/2018 e 16/12/2018 (órbita ponto 223/81); 15/02/2018, 26/08/2018 e 16/12/2018 (órbita ponto 224/81); e 13/01/2017, 25/08/2017 e 15/12/2017 (órbita ponto 221/81). As imagens foram adquiridas no Serviço Geológico dos Estados Unidos (USGS), e foram consideradas as bandas do espectro óptico (B2, B3, B4, B5, B6 e B7) em reflectância de superfície (SR), resolução espacial de 30 metros e a banda termal em temperatura de brilho $(\mathrm{Tb})$, com a resolução espacial reamostrada para 30 metros.

\subsection{Métodos}

Os procedimentos metodológicos seguiram três etapas: Etapa 1 - pré-processamento das imagens; Etapa 2 - coleta de amostras de treinamento; Etapa 3 - classificação supervisionada e análise de acurácia.

\subsubsection{Etapa 1}

As imagens termais em Temperatura de Brilho $(\mathrm{Tb})$ passaram pelo processo de correção de emissividade (Equação 1) com auxílio do software QGIS e metodologia implementada por Ndossi e Avdan (2016). As imagens de emissividade consideram os valores de NDVI (Tabela 1), conforme metodologia utilizada em diferentes pesquisas (Van De Griend \& Owe, 1993; Valor \& Caselles, 1996; Sobrino, Jiménez-Muñoz, \& Paolini, 2004; Zhang, Wang, \& Li, 2006).

$$
\mathrm{TS}=\mathrm{Tb} /\left(1+(\lambda \mathrm{Tb} / \alpha)^{*} \ln \varepsilon\right) \quad \text { Equação } 1
$$

$\mathrm{TS}-$ Temperatura de Superfície, $\mathrm{Tb}=$ temperatura de brilho registrada pelo sensor em Kelvin; $\lambda=$ comprimento de onda médio do infravermelho termal; $\alpha=\mathrm{hc} / \mathrm{K}=1.438 \times 10$ $2 \mathrm{~m} \mathrm{~K}(\mathrm{~K}=$ constante de Stefan-Boltzman $(1.38 \times 10-23 \mathrm{~J} / \mathrm{K}) ; \mathrm{h}=$ constante de Planck $(6.28 \times 10-34 \mathrm{~J} / \mathrm{s}) ; \mathrm{c}=$ velocidade da luz $(2.998 \times 108 \mathrm{~m} / \mathrm{s}))$. 
Tabela 1. Relação NDVI e Emissividade

\begin{tabular}{|c|c|}
\hline Valores NDVI & Valores Emissividade \\
\hline NDVI $<-0.185$ & 0.995 \\
\hline$-0.185 \leq$ NDVI $<0.157$ & 0.985 \\
\hline $0.157 \leq \mathrm{NDVI} \leq 0.727$ & $1.009+0.04 \times \ln (\mathrm{NDVI})$ \\
\hline $\mathrm{NDVI}>0.727$ & 0.990 \\
\hline
\end{tabular}

Fonte: adaptado Ndossi e Avdan (2016).

\subsubsection{Etapa 2}

A coleta de amostras de treinamento abordou as classes área de cultivo (AC), campo (CA) e vegetação arbórea (VA). Todas as coberturas que não se enquadravam nessas classes foram atribuídos à classe "outros" (não computada nas análises). Uma máscara de corpos hídricos disponível na Plataforma TerraBrasilis foi aplicada, estas áreas não foram consideradas na classificação. No total, foram coletados 3000 pixels de treinamento (1000 pixels para cada classe).

\subsubsection{Etapa 3}

Neste estudo foram realizadas classificações supervisionadas com o algoritmo paramétrico Máxima Verossimilhança - Maxver, um dos algoritmos mais utilizados em pesquisas de sensoriamento remote (Silveira et al., 2020). Para fins de comparação, duas classificações foram realizadas: classificação 1 - somente as bandas SR e classificação 2 - combinação das bandas SR e bandas Termal.

A análise da acurácia das classificações foi realizada a partir de 450 pontos distribuídos aleatoriamente nas células de estudo, sendo cada ponto interpretado por diferentes intérpretes para garantir maior confiabilidade nos pontos de referência. A interpretação contou com composições coloridas das imagens Landsat 8 para diferentes estações do ano e imagens de alta resolução disponíveis no Google Earth com datas próximas das imagens utilizadas para as classificações. Os pontos também foram conferidos com os dados de desmatamento 2016/2018 e disponibilizados na plataforma TerraBrasilis do INPE (Assis et al., 2019, Almeida, 2020).

A partir das imagens classificadas e os pontos de referência, foram calculadas as matrizes de erros para cada classificação, e assim calculadas a Exatidão Global (EG), Exatidão do Produtor (EP) e Exatidão do Usuário (EU). Desta forma, foi possível verificar o desempenho do classificador e a contribuição da banda termal nas classificações.

\section{Resultados e Discussão}

A figura 2 representa as características das classes selecionadas no bioma Pampa. Na imagem termal de dezembro verificou-se que as classes estudadas apresentaram diferenças de TS (Figura 2 a), as áreas de cultivo com os valores de TS mais elevados e a vegetação arbórea com a menor média de TS. 
a)

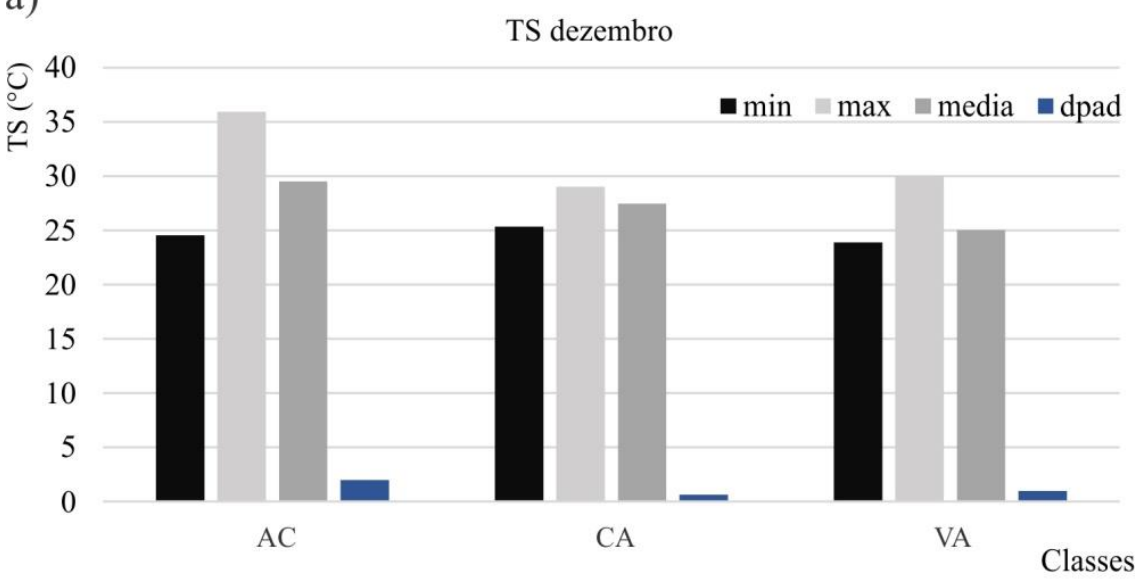

b)

Composições coloridas imagem Landsat
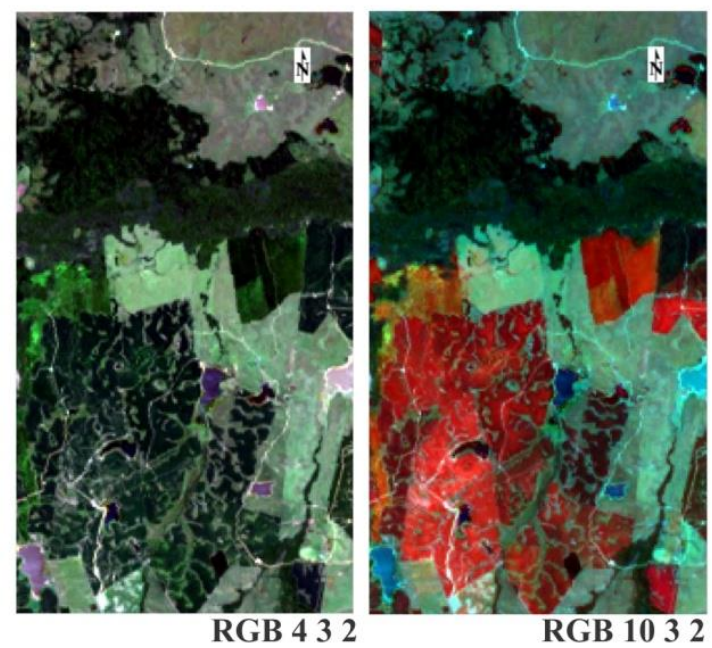

c)
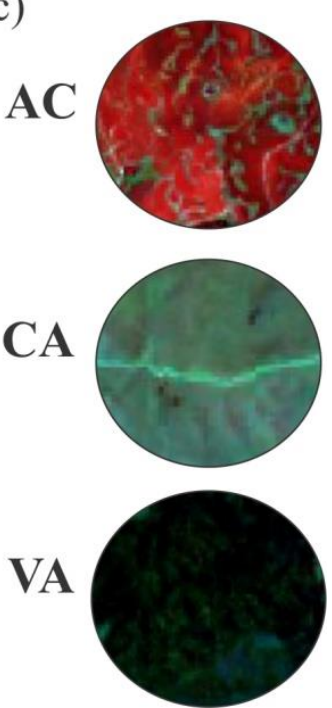

Figura 2 Características das classes área de cultivo (AC), campo (CA) e vegetação arbórea (VA): a) estatísticas da TS para cada classe na imagem 16/12/2018; b) composições coloridas identificando cada classe; c) destaque para as classes AC, CA e VA.

A adição da banda termal na composição colorida falsa cor RGB 1032 (Figura 2 b) possibilitou maior diferenciação entre as classes (Figura 2 c), e maior destaque para a classe AC. Na composição colorida cor verdadeira RGB 432 da imagem de fevereiro, as classes área de cultivo e vegetação arbórea não foram facilmente diferenciadas, o que destaca a contribuição da banda termal e de diferente estação do ano para análise qualitativa das imagens. O padrão de cobertura das áreas agrícolas muda frequentemente (Blaschke, et al., 2000), portanto, em época de alto vigor vegetativo (fevereiro) a temperatura da superfície é inferior à temperatura da época de solo exposto (dezembro), contribuindo para o maior destaque quando utilizada a banda termal na composição de bandas. Eisavi et al. (2015) destacam que a utilização de imagens multitemporais e termais tem potencial de melhorar a eficiência da classificação da cobertura da terra.

Um exemplo dos mapas produzidos neste estudo é apresentado na Figura 3, sendo possível verificar as diferenças das áreas classificadas. Na classificação 1 foram utilizadas 
somente as bandas em reflectância de superfície (SR), e a classificação 2 abordou as bandas SR e TS.

O desempenho das classificações foi avaliado conforme o cálculo da Exatidão Global (EG), Exatidão do Produtor (EP) e Exatidão do Usuário (ES). A EG das classificações teve variação de $80 \%$ (classificação 1) a $83 \%$ (classificação 2), ou um acréscimo de 3\% na acurácia da classificação com a inclusão da banda termal.

Ao utilizar imagens Landsat 8 de diferentes estações do ano, Zhao et al. 2019 também avaliaram a classificação de imagens com a inclusão da banda termal. Os autores concluíram que a adição das imagens termais aumentou a acurácia dos mapas de cobertura da terra, apresentando um incremento de $1 \%$ nos mapas classificados.

Ao analisar os padrões de cobertura do solo, Sun e Schulz (2015) também destacaram a eficácia das bandas termais. Os autores constataram que ao combinar a banda termal (Landsat 8 ) às bandas do visível e infarvermelho próximo, ocorreu um aumento da acurácia da classificação de $3 \%$ a $6 \%$.

O estudo realizado por Eisavi et al. (2015) apontou as seguintes exatidões globais para diferentes cenários: ao considerar apenas bandas ópticas EG de 86,48\%; apenas banda termal EG de 82,26\%; e a combinação de bandas ópticas com termal apresentou uma EG de 90,63\%. Assim, pode-se confirmar que a banda termal sozinha não tem a eficácia de aumentar a EG da classificação, mas a combinação com as demais bandas do espectro pode contribuir para o aumento da acurácia nas classificações de cobertura da terra.

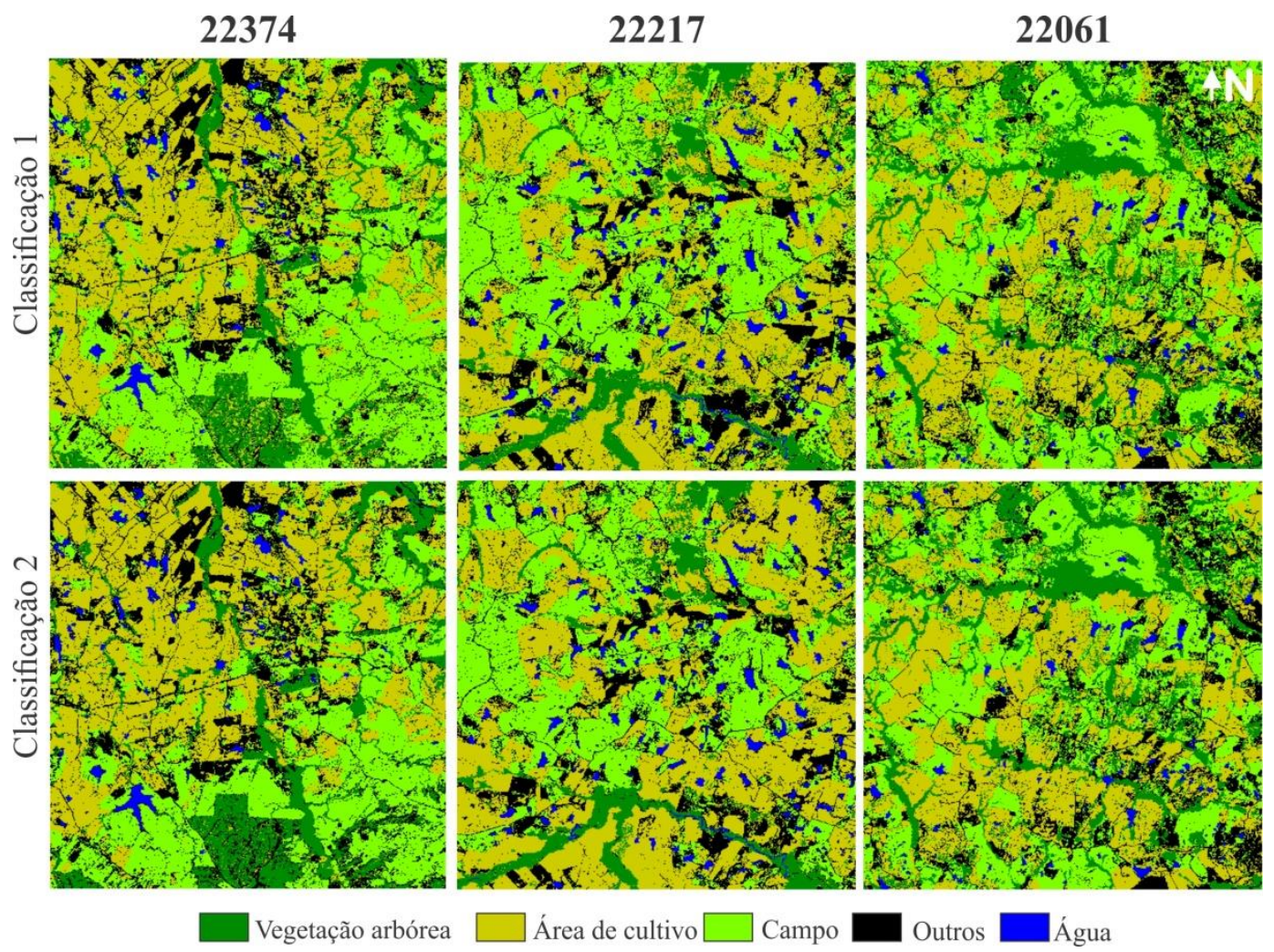

Figura 3. Exemplo de células classificadas com banda termal (Classificação 2) e sem a banda termal(Classificação 1). 
A tabela 2 mostra o desempenho da classificação para cada classe a partir da EP e EU. Foi possível verificar que a média de EP variou de 79,67\% (classificação 1) a 83,43\% (classificação 2) e a EU de 79,32 \% a 82,43\%. A inclusão da banda termal na classificação garantiu um aumento da EP e EU para as três classes analisadas. Ao analisar diferentes cenários de classificação com a banda 10 Landsat 8, Eisavi et al. (2015) verificaram uma variação de EP média de 77,48\% para classificação sem a banda termal e 80,12\% para classificação com a inclusão da banda termal. Os valores de EU encontrados foram de $77,14 \%$ (sem termal) e 78,61 (com termal).

Tabela 2 - Exatidões do produtor e usuário para as classes área de cultivo (AC), campo (CA) e vegetação arbórea (VA) nas classificações

\begin{tabular}{|l|c|c|c|c|c|c|c|c|}
\hline & \multicolumn{4}{|c|}{ Exatidão do produtor (\%) } & \multicolumn{4}{c|}{ Exatidão do usuário (\%) } \\
\cline { 2 - 9 } & AC & CA & VA & Média & AC & CA & VA & Média \\
\hline Classificação 1 & 77,83 & 84,07 & 77,10 & 79,67 & 84,46 & 76,41 & 77,10 & 79,32 \\
\hline Classificação 2 & 79,68 & 88,50 & 81,54 & 83,24 & 89,26 & 78,33 & 79,70 & 82,43 \\
\hline
\end{tabular}

*Classificação 1 -somente bandas SR; Classificação 2 - bandas SR e Termal.

A classe campo apresentou a maior EP $(88,5 \%)$, ou seja, $88,5 \%$ das áreas de campo foram corretamente classificadas com a inclusão da banda termal, enquanto a classificação 1 (somente bandas SR) apresentou um EP de 84,07\% para a classe supracitada. A classe VA apresentou um aumento de 4,44\% na EP e 2,6\% na EU. As áreas de cultivo apresentaram um incremento de $1,85 \%$ na EP e $4,8 \%$ na EU. Resultados semelhantes foram encontrados por Zhao et al. (2019), com incremento na EP 1\% e EU de até $2 \%$ para áreas de cultivo quando considerada a banda termal na classificação.

\section{Considerações finais}

A realização do trabalho permitiu destacar as seguintes afirmações:

- A inclusão da banda termal aumentou a acurácia dos mapas classificados, com incremento de $3 \%$, valor dentro dos resultados encontrados em diferentes pesquisas internacionais.

- A adição da banda termal em composições coloridas falsa-cor possibilitou a melhor diferenciação entre as classes. Então, a banda termal também contribui de forma qualitativa para a interpretação e classificação de imagens.

- A combinação de bandas de diferentes estações do ano possibilita a melhoria da classificação, principalmente, para a interpretação das imagens.

- Estão sendo realizados outros processamentos com diferentes classificadores para refinar as classificações da cobertura da terra para o bioma Pampa, considerando imagens de diferentes datas em séries temporais mais extensas. 


\section{Agradecimentos}

Este artigo é um dos resultados do Projeto CNPq 444418/2018-0 (Monitoramento dos biomas brasileiros por satélite - Construção de novas capacidades), que disponibilizou bolsas para as duas primeiras autoras.

\section{Referências}

Almeida, C.A.; Valeriano, D.M; Maurano, L.; Vinhas, L.; Fonseca, L.M.G.; Silva, D.; Santos, C.P.F; Martins, F. S. R. V.; Lara, F.C.B.; Maia, J.S.; Profeta, E.R.; Santos, L.O.; Santos, F.C.O; Ribeiro, V. 'Deforestation monitoring in different Brazilian Biomes: challenges and lessons.'In: ISPRS Annals of the Photogrammetry, Remote Sensing and Spatial Information Sciences, Volume IV-3/W2-2020, 2020, Santiago, Chile.

Assis, L. F. F. G, Ferreira, K. R., Vinhas, L., Maurano, L., Almeida, C., Carvalho, A., Camargo, C. 2019. TerraBrasilis: A Spatial Data Analytics Infrastructure for LargeScale Thematic Mapping. ISPRS International Journal of Geo-Information, 8(11), 513. doi: 10.3390/ijgi8110513

Blaschke, T.; Lang, S.; Lorup, E.; Strobl, J.; Zeil,P.; "Object-oriented image processing in an integrated GIS/remote sensing environment and perspectives for environmental applications". In: Cremers, A. B. \& Greve, K. (Hrsg). (2000)

Eisavi, V., Homayouni, S., Yazdi, A. M., \& Alimohammadi, A. (2015). Land cover mapping based on random forest classification of multitemporal spectral and thermal images. Environmental Monitoring and Assessment, 187(5), 1-14. https://doi.org/10.1007/s10661-015-4489-3

Embrapa. Nosso Pampa desconhecido. Porto Alegre: Fundação Zoobotânica do Rio Grande do Sul, 2016. In: Chomenko, 1.; Bencke, G. A. (Org.) Capítulo em livro técnico (INFOTECA-E). 2016.

MMA (Ministério do Meio Ambiente). "Pampa". Folder Pampa. (2019) Accessed 15 Abril 2020. https://www.mma.gov.br/biomas/pampa

Nabinger, C.; Ferreira, E.T.; Freitas, A.K.; Carvalho, P.C.F.; Sant'anna, D.M. Produção animal em campo nativo: aplicações de resultados de pesquisa. In: Pillar, V.P.; Müller, S.C.; Castilhos, Z.M.S.; Jacques, A.V.A. (Org.). Campos sulinos: conservação e uso sustentável da biodiversidade. Brasília: Ministério do Meio Ambiente - MMA, p. 175198. 2009

Ndossi, M. I., And Avdan, U. 2016. Application of open source coding technologies in the production of Land Surface Temperature (LST) maps from Landsat: A PyQGIS plugin. Remote Sensing, 8 (5), 1 - 31. doi: 10.3390/rs8050413

Rodríguez-Galiano, V. F., Ghimire, B., Pardo-Igúzquiza, E., Chica-Olmo, M., \& Congalton, R. G. "Incorporating the downscaled Landsat TM thermal band in landcover classification using random forest". Photogrammetric Engineering and Remote Sensing, 78(2), 129-137 (2012). doi: 10.14358/PERS.78.2.129 
SILVEIRA, A.H de M.; SILVA, F.M,; HADAD, R. M.; LIBÓRIO, M.P. Alicações, preferências e comparações entre métodos de classificação supervisionada: o caso de Natal/RN. Ra'e Ga. Curitiba, v.47, n.1, p. 120-135.

Sobrino, J. A., Jiménez-Muñoz, J. C., And Paolini, L. 2004. Land surface temperature retrieval from LANDSAT TM 5. Remote Sensing of Environment, 90(4), 434-440. doi: $10.1016 /$ j.rse.2004.02.003

Sun, L.; Schulz, K. The Improvement of Land Cover Classification by Thermal Remote Sensing. Remote Sensing, 7, 8368 - 8390. doi:10.3390/rs70708368

Valor, E., And Caselles, V. 1996. Mapping land surface emissivity from NDVI: Application to European, African, and South American areas. Remote Sensing of Environment, 57(3), 167-184. doi: 10.1016/0034-4257(96)00039-9

Van De Griend, A. A., and Owen, M. 1993. On the relationship between thermal emissivity and the normalized difference vegetation index for natural surfaces. International Journal of Remote Sensing, 14(6), 1119-1131. doi: $10.1080 / 01431169308904400$

Zhang, J., Wang, Y., And Li, Y. 2006. A C++ program for retrieving land surface temperature from the data of Landsat TM/ETM+ band6. Computers and Geosciences, 32(10), 1796-1805. doi: 10.1016/j.cageo.2006.05.001

Zhao, J., Yu, L., Xu, Y., Ren, H., Huang, X., \& Gong, P. (2019). Exploring the addition of Landsat 8 thermal band in land-cover mapping. International Journal of Remote Sensing, 40(12), 4544-4559. https://doi.org/10.1080/01431161.2019.1569281. 